\title{
Sexual Behavior Research on a Cohort of Gay Men, 1984-1990: Can We Predict How Men Will Respond to Interventions?
}

\author{
David G. Ostrow, M.D., Ph.D., ${ }^{1,3}$ Eugenio Beltran, D.D.S., M.P.H., ${ }^{2}$ \\ and Jill Joseph, Ph.D. ${ }^{1}$
}

In 1984, over 1000 gay and bisexual men volunteered to participate in both the Chicago Multicenter AIDS Cohort Study (MACS) and a companion psychosocial study, the Coping and Change Study (CCS). Participants in the semiannual Chicago MACS/CCS evaluations comprise the largest cohort of high-risk men under continuous medical, behavioral, and psychosocial observation. Chicago MACS/CCS researchers prospectively chart the sexual behavior change patterns of the cohort and relate those behavioral changes to psychosocial correlates and actual HIV infection risk. This report summarizes the behavioral natural history of the Chicago MACS/CCS cohort from 1984 to 1990, focusing on receptive anal sex practices and use patterns for alcohol and the most frequently used recreational drugs. As these are prospective observational and not controlled intervention studies, psychosocial correlates of sexual behavior change by members of the cohort are suggestive of factors influencing behavior change rather than indicative of causal relationships. However, the voluntary availability to participants in the Chicago MACS/CCS of $H I V-1$ antibody test results beginning in late 1985 provided the opportunity to examine whether demographic, psychosocial, or behavioral factors were indicators of sexual behavior change following disclosure and counseling about

Paper presented at the 1991 Annual Meeting of the International Academy of Sex Research, Barrie, Ontario, Canada.

${ }_{1}^{1}$ Department of Epidemiology, University of Michigan School of Public Health.

${ }^{2}$ Formerly Department of Dental Public Health, University of Michigan School of Public Health. Currently Dental Public Health, Center for Prevention, Centers for Disease Control, Atlanta, Georgia.

${ }^{3}$ To whom correspondence should be addressed at Center for AIDS Intervention Research, Medical College of Wisconsin, 1201 N. Prospect Ave., Milwaukee, Wisconsin 53202. 
$H I V-1$ serostatus. Recommendations for promotion and maintenance of safer sexual behavior for the long run, and limitations in the generalizability of these findings to the much more diverse populations of men who have sex with other men conclude this article.

KEY WORDS: anal sex; behavior change; psychosocial predictors; HIV testing; substance use; homosexual men.

\section{INTRODUCTION}

In 1984, over 1000 men volunteered to take part in the Chicago cohort of the Multicenter AIDS Cohort Study (MACS), a NIAID-sponsored long-term study of the epidemiology, natural history, and immunovirology of AIDS and HIV infection (Kaslow et al., 1987). Simultaneously, over 95\% of the participants in the Chicago MACS have participated in semiannual behavioral, psychosocial, and functional assessments as part of their participation in the NIMH-sponsored Coping \& Change Study (CCS). These men comprise the largest group of homosexual/bisexual men at risk for AIDS or HIV under continuous biopsychosocial observation, and have permitted Chicago MACS/CCS investigators to prospectively follow the behavioral adaptation of these men and relate any sexual behavior change to both psychosocial correlates and actual HIV infection risk (Joseph et al., 1984; Emmons et al., 1986; Ostrow et al., 1989b). These observational studies have helped to elucidate the major behavioral adaptations that homosexual/bisexual men in Chicago have undertaken to reduce their chances of acquiring or transmitting HIV infection. Chicago MACS/CCS investigators have described the high rate of behavioral relapse to less safe sexual practices and identified specific demographic, behavioral, and psychosocial correlates for both the adoption of safer sex and relapse to unsafe sexual practices; shown that aggregate sexual behavior change patterns during the first 2-3 years of the study (1984-1986) were dramatically in the direction of less HIV risk and were independent of whether individual participants were informed of their HIV-1 antibody status; and demonstrated the general association of recreational drug use with unsafe sexual activities and relapse to unprotected anal sex in association with amyl nitrite use ("poppers") specifically.

One major purpose for undertaking a long-term set of observational studies such as the Chicago MACS/CCS is the importance of being able to observe and understand long-term behavioral changes among at-risk communities. Previously, Chicago MACS/CCS investigators have analyzed 1- or 2-year intervals of sexual behavior change patterns among members of the cohort and/or related specific theoretical and empirical psychosocial 
constructs to the observed behavior changes (Emmons et al., 1986; Joseph et al., 1987; Montgomery et al., 1989; Ostrow et al., 1989a; Adib et al., 1991). Such efforts, however, have been limited in their ability to "explain" behavioral change through specific psychosocial or environmental factors, frequently pointing up the importance of preexisting behavior patterns in determining future behaviors. For example, our attempts to apply the Health Belief Model (HBM) constructs to behavioral change during the first 2 years of the Chicago MACS/CCS showed that individual and combined HBM measures added, at most, 10-20\% to the proportion of change explained by baseline behavioral and sociodemographic factors (Montgomery et al., 1989). Even more frustrating has been our inability to discern the behavioral effects of major social, medical, or mass educational events among the cohort in general (Ostrow et al., 1988; 1989b).

There are several methodological explanations for our previous inability to observe significant effects of psychosocial factors on sexual behavior change in this cohort. The first is that these are observational studies and do not have a controlled intervention design that would permit the discernment of specific intervention effects. If the net effect of any environmental or psychosocial occurrence were to be an approximately equal number of persons changing in opposite directions in terms of sexual HIV transmission risk, then we would observe no "net" effect of the circumstance regardless of the amount and magnitude of individual behavior change responses. In fact, most if not all observational studies of behavioral responses to HIV have assumed that the individual members of the group or cohort under observation will respond in a uniform manner to those extant behavioral change influences in the group or society. However, there has been a growing recognition among AIDS behavioral researchers that individual responses to the threat of AIDS are likely to be different depending on the individual's stage of risk reduction, their previous behavioral response patterns, their physical and mental health, ethnicity, culture, and social milieu (Ostrow, 1989a, 1989b, 1990; Mays et al., 1989; Johnson et al., 1990). In accordance with recognition of the possibility of diverse behavioral responses, we have begun to take a longer term and individually based look at the sexual behavior change patterns of the men participating in the Chicago MACS/CCS studies. When psychosocial and environmental correlates of multiple behavioral responses are studied, we can identify more robust influences on specific behavioral change patterns, allowing us to identify men at risk of increasing their sexual risk behavior following HIV antibody testing and counseling (Ostrow et al., 1989a; Beltran et al., 1993). Discussion of the implications of these findings to future HIV prevention intervention efforts as well as the limits of their generalizability to 
other populations of men at homosexual risk of $\mathrm{HIV}$ infection conclude this article.

\section{METHODS}

\section{Study Population}

The participants of the Chicago MACS/CCS have been providing information and laboratory specimens concerning their demographic, physical, epidemiological, virological, immunological, behavioral, and psychosocial characteristics every 6 months since 1984 (Kaslow et al., 1987; Joseph et al., 1984). Most of the men were recruited to participate in the Chicago cohort of the MACS through a gay community-based sexually transmitted disease clinic, the Howard Brown Health Clinic (HBHC); approximately $15 \%$ were recruited through the Infectious Disease or Immunology Clinics of Northwestern Memorial Hospital in downtown Chicago. The majority (93\%) of the original Chicago MACS/CCS participants were white; while efforts have been ongoing within the Chicago MACS to recruit nonwhite participants, members of the supplemental cohort were not assessed before 1987 and were not given the option of whether or not they received their HIV antibody test results and so are not included in these analyses. A reliable laboratory technique for HIV-1 antibody detection became available only in late 1985 (Weiss et al., 1985), and its results first made available to the MACS cohorts beginning with their fourth semiannual visit. In contrast to later policy recommendations regarding HIV antibody testing and disclosure (McCarthy, 1988), disclosure of HIV test results to original Chicago MACS cohort members was and still is entirely voluntary. We have previously taken advantage of the fact that only a minority of Chicago MACS/CCS participants chose to learn their HIV serostatus during the first 2 years that test results and posttest counseling were available to demonstrate the lack of any overall sexual behavior effects of HIV antibody serostatus disclosure (Ostrow et al., 1989b).

In the following report we describe the overall patterns of receptive anal sexual practices and substance use behaviors as well as demonstrate the behavioral, psychosocial, and demographic correlates of the various patterns of anal sex behavioral change observed during the interval of mid1985 to mid-1987. During that interval, 1020 men completed both a CCS self-administered questionnaire (SAQ) and a MACS evaluation on at least one semiannual MACS visit, and 635 men participated in the entire set of four Chicago MACS/CCS assessments, comprising a Visit Three Through Six (V3-6) panel sample. Comparing the V3-6 panel members to the entire 
cohort of 1020 men, panel members were older ( 34.9 vs. 33.2 years old), had higher incomes $(\$ 23,700$ vs. $\$ 21,300)$ and were more educated $(16.4$ vs. 15.6 years) than those excluded due to missed visits. Of the 635 men assessed at all four V3-6 semiannual intervals, 177 (28\%) requested and received their HIV-1 antibody test results (disclosed group) while 458 (72\%) neither requested nor received their test results (nondisclosed group) between December 1985 and June 1987. A random allocation procedure was used to select comparison subgroups of nondisclosed men who completed both MACS and CCS evaluations for each of the semiannual pairs of MACS visits at which the disclosed men had their pre- and postdisclosure evaluations.

For the behavioral change descriptions that begin this report, we utilized the maximum number of men participating in both the Chicago MACS and the CCS available for analysis at each visit (maximum $N=1006$ ): 514 men $(51 \%)$ were seronegative throughout the study period (V1-12); 405 men $(40 \%)$ were seropositive at entry into the study; and 87 men (9\%) seroconverted from negative to positive between V1 and V12. The total cohort did not differ from either V1-9 or V4-12 subsamples in terms of education or income levels, likelihood to seroconvert, or levels of receptive anal sex or substance use at any time in the study.

\section{Outcome Measures}

The primary sexual behavior outcome measure used in this study is receptive anal sex (RAS), the sexual practice associated with the highest risk of HIV infection among men in the MACS (Kingsley et al., 1987; Detels et al., 1989). Men were instructed to fill out the CCS SAQ 2 weeks following each MACS visit and asked about the number of each type of male sexual partners (steady or primary, regular, casual, or anonymous) with whom they had RAS during the last month. On the basis of their answers to these questions and whether or not they reported the use of condoms with any or all of their sexual partners, the men were then classified into one of four RAS risk levels:

1. No risk: No reported sexual intercourse (celibacy) or partners.

2. Low risk: Sexual partner(s) reported and either no RAS or RAS only with primary partner and consistent condom use.

3. Modified high risk: Either consistently used condoms during RAS with multiple partners or limited unprotected RAS to single primary partner.

4. High risk: Reported unprotected RAS with multiple partners. 
We have previously reported the hierarchical HIV infection risk associated with this four-level RAS risk index among initially seronegative men in the Chicago MACS/CCS (Joseph et al., 1987; 1989; Ostrow 1989a) and the overall MACS (Ostrow et al., 1990). For purposes of categorizing the pre- to postdisclosure change in sexual risk, the four-level RAS risk index was dichotomized into either low (no or low risk levels 1 and 2) or high (modified high and high risk levels 3 and 4) risk for each visit. V3-6 panel participants were then further categorized into four patterns of sexual behavior change across the single 6-month interval that included their serostatus disclosure or, for nondisclosed men, the matched 6-month assessment interval: (i) stable high risk (high RAS risk level both before and after the pre- to postdisclosure interval), (ii) increasing risk (low RAS risk pre- to high risk postdisclosure), (iii) decreasing risk (high pre- to low postdisclosure risk), and (iv) stable low risk.

Alcohol and drug use measures were collected as part of the faceto-face MACS interviews conducted at each semiannual visit. At baseline, questions were asked to determine the frequency (but not quantity) of use of each of 10 classes of recreational drugs during the past 2 years and during the past 6 months. For alcoholic beverages, both frequency and quantity were recorded. At all follow-up visits, the questions were repeated for the period since the last visit (usually 6 months). From these data, summary substance use measures were created as follows: (i) frequency of use of each drug and a summary measure of all drug use reported for the prior 6 months and (ii) number of alcoholic beverages consumed per month, further classified into low use (up to 12 drinks per month), moderate use (between 13 and 59 drinks per month), or heavy use (greater than 59 drinks per month) (Clark and Midanik, 1982).

\section{Psychosocial Predictors of Sexual Behavior Change Associated with HIV-1 Antibody Disclosure}

A set of variables were assessed using one-way analysis of variance (ANOVA) and multivariate logistic regression (Cox, 1972) for this ability to predict, at the time of the predisclosure CCS SAQ, the pre- to postdisclosure pattern of RAS behavior change, according to the above typology. The predictors examined included basic demographic information - such as age, race, education, and income levels - and a set of previously described psychosocial measures (Joseph et al., 1984, 1987) including mental health [Brief Symptom Inventory (BSI), Derogatis et al., 1974], perceived social supports (O'Brien et al., 1993), and coping strategies (Joseph et al., 1989). Predictors found to differ significantly by ANOVA between the four behavior change patterns 
were tested in hierarchical logistical models (comparing each favorable and adverse behavioral outcome pair) and were either left in or deleted from the models depending on either the significance on the Wald's test (beta coefficient divided by its standard error) or the likelihood ratio test (Kleinbaum et al., 1982) compared to a chi-squared distribution (Harrell, 1986). To control for the effect of HIV-1 serostatus disclosure without losing statistical power, all participants (disclosed and matched nondisclosed) were included in the logistical analyses and, using standard analytical techniques (Lewis-Beck, 1980 ), each predictor variable in the model was multiplied by a disclosure factor $(1=$ nondisclosed, $2=$ disclosed $)$.

\section{RESULTS}

\section{Sexual Behavior Patterns, 1984-1990}

Examination of the cohort according to the sexual risk index (Fig. 1) reveals significant decreases in unsafe RAS practices between V1 and V3, with lesser overall risk reduction occurring between V3 and V7 and a plateau in aggregate risk reduction thereafter. When RAS behavioral practices between baseline and V12 were examined separately for seropositive and seronegative men, there were significant differences related to serostatus only for the first two or three semiannual assessment periods-seropositive men began participation in the Chicago MACS/CCS at higher average levels of sexual risk behavior, made greater initial reductions in RAS, and followed the same behavior change curves as the initially seronegative men from V3 onwards (data not shown). These sexual behavior change profiles were paralleled by dramatic reductions in the seroconversion rates for initially seronegative members of the cohort, dropping from approximately seven seroconversions/100 person-years of observation between V1 and V2, to zero to one seroconversions by the middle of the observation period (V4-5 to V8-9 intervals). However, the apparent stabilization in rates of unprotected RAS among cohort members from V5 onward seen in Fig. 1 obscures the high rates of lapse/relapse to unprotected RAS observed when individual patterns of RAS behavior change are examined (Adib et al., 1992) and which have been accompanied by an increase in seroconversion rates among the men who were still seronegative as of V9 to as high as four seroconversions/100 person-years of observation between V10 and V12. (Kingsley $e t$ al., 1991). These findings highlight the pitfalls of examining only aggregate patterns of sexual risk behaviors rather than individual patterns of behavior change in a cohort with as much movement in both di- 


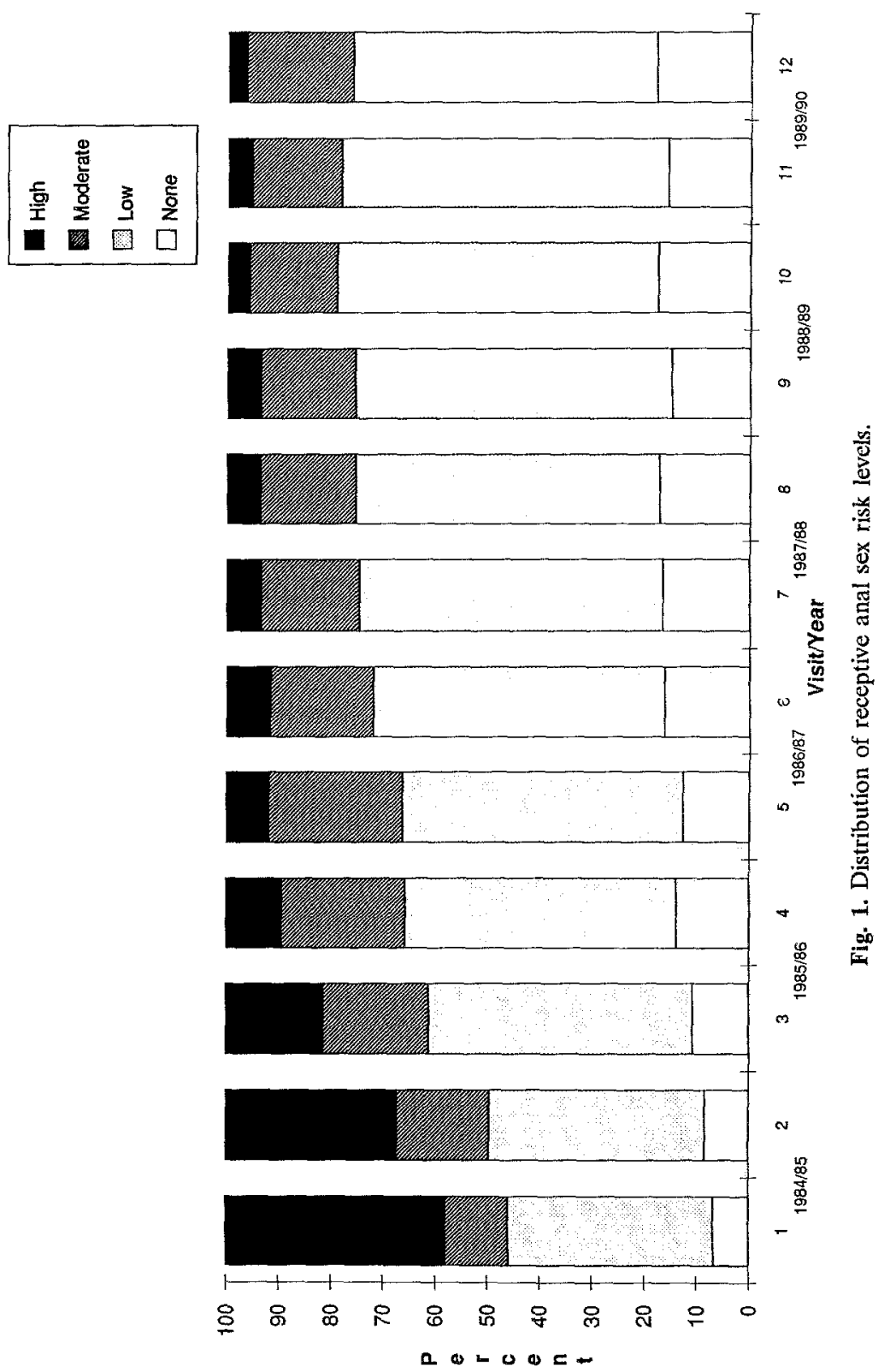


rections of sexual behavior change as the Chicago MACS/CCS (Joseph et al., 1990).

Overall patterns of sexual behavior change in this cohort do illustrate the relative popularity of the three alternative risk reducing behavioral changes, namely, adoption of celibacy, consistent use of condoms, and avoidance of RAS. The rate of reported celibacy has remained relatively unchanged, between 11-18\% of the cohort reporting no sexual partners during the last month at any time between 1984 and 1990 . Only 10-15\% of the men initially reporting unprotected RAS have adopted consistent condom use or restriction of unprotected RAS to a single sexual partner at any time since the first two visits, while a majority of the cohort members report avoidance of RAS as their major behavioral change. However, both consistent condom use and avoidance of RAS are behavioral changes highly prone to lapses of unprotected RAS, at rates of up to one third of the men at risk of behavioral relapse per year in this cohort (Adib et al., 1992).

\section{Alcohol and Recreational Drug Use Patterns, 1984-1990}

Alcohol was reportedly used by $90-95 \%$ of participants at each visit (Fig. 2). The proportion of men who reported light (less than 13 drinks/month) and moderate use (13-59 drinks/month) remained fairly constant over time, ranging from 28 to $40 \%$ and 35 to $45 \%$, respectively. The proportion of heavy alcohol users (more than 59 drinks/month) dropped sharply from $28 \%$ of the cohort at baseline to $19 \%$ at V2 and decreased gradually until reaching a plateau at $12-15 \%$ between V6 and V12 (Fig. 2).

In contrast to the relative stability of alcohol use patterns in the cohort over the 7 year observation period, there were dramatic reductions in the reported number and frequency of recreational drugs used by members of the cohort between study entry (1984) and V6 or V7 (1987). Marijuana and poppers were the drugs most frequently used by participants throughout the study period. Participants reported a decrease in use of both drugs from $70 \%$ at baseline to $45-50 \%$ at visits $5 / 6$ and staying relatively constant $(43-46 \%)$ thereafter. Cocaine was consistently found to be the third most frequently used drug; one third of the cohort reported its use at baseline, declining to $16 \%$ by visit 6 , and staying constant (15-17\%) thereafter. Crack use was only assessed beginning with V10 and was reportedly used only by a handful of men at V12. MDA, psychedelics, downers, and uppers were used by 15 to $19 \%$ of participants at baseline and falling to between 4-5\% usage by visit 5 . The last group of recreational drugs assessed, including ethyl chloride, opiates, and other drugs, were used by less than $5 \%$ 

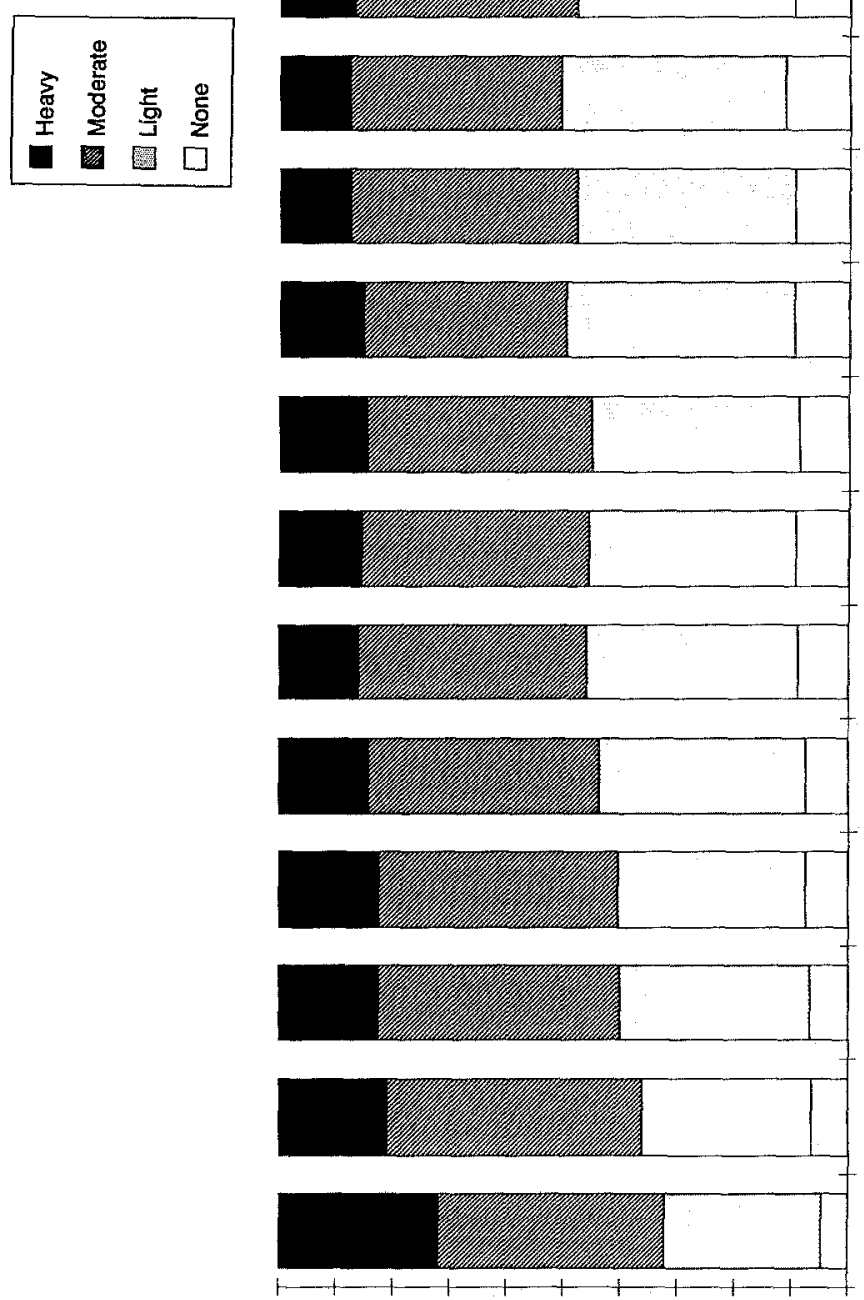

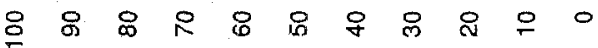

ด.

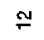<smiles>[B]=[BH2-]</smiles>

$=$

우

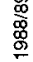

$\sigma$

$\frac{\dot{2}}{0}$

$\infty$

怘兽焉

N 는

$\sum_{\substack{0 \\ 0}}^{\mathbb{8}}$

$\omega \stackrel{\frac{0}{8}}{\frac{8}{0}}$

鱼

\%

$\infty \quad$ 运

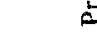

悤 粮

N

㽞
总
。 
of the cohort at any time, while drug use by needle was reported by less than $1 \%$ of the men at any time.

Figures 3-5 illustrate the patterns of use of the three most popular recreational drugs and cigarette smoking stratified by the HIV-1 serological status of participants. Seropositive men (Fig. 4) reported higher initial proportionate use of all drug categories and greater residual drug use levels at later visits than did seronegative men (Fig. 3). Patterns of drug use for seroconverters (Fig. 5) resembled those of seropositive men, except that seroconverters had higher initial levels of popper use and appear to have had an increase in the proportion of men using all three of the popular drugs during the V9-12 interval, the same period as when the second burst of new seroconversions was observed in the cohort (Kingsley et al., 1991).

\section{Prediction of Sexual Behavior Change Patterns Associated with HIV-1 Test Disclosure}

Figures 6-9 show the mean predisclosure demographic, mental health, coping, and social support measures, respectively, for each of the four sexual behavior change subgroups (stable low risk, decreasing risk, stable high risk, and increasing risk) of the 177 men requesting their HIV -1 status during the V3-6 period. The most consistent feature of these subgroup comparisons are the extreme values of the demographic and psychosocial measures for the subgroup whose RAS risk increased from the predisclosure to postdisclosure visit (right-hand columns). Comparing the

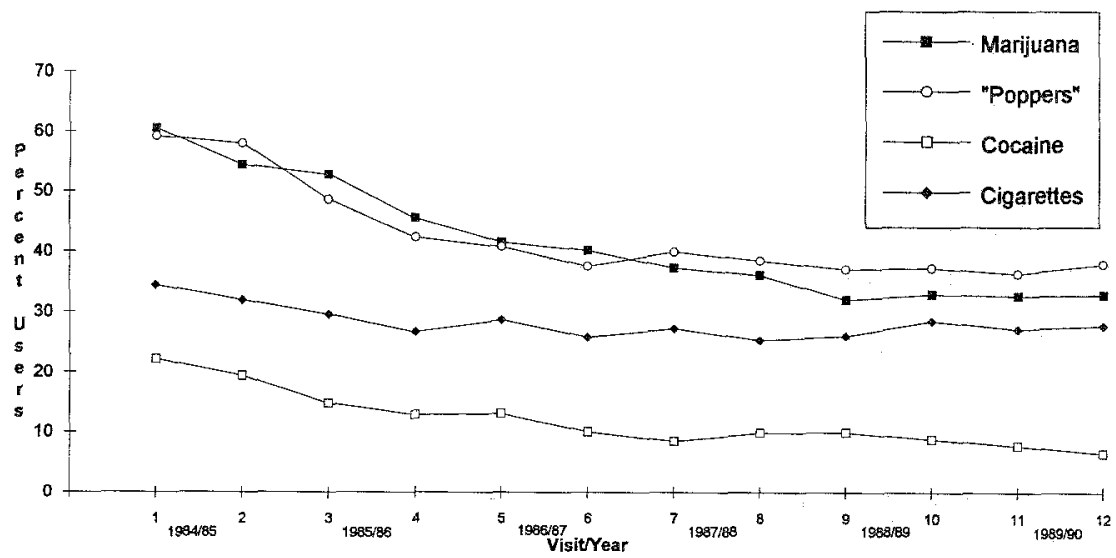

Fig. 3. Prevalence of most frequently used substances among seronegative men. 


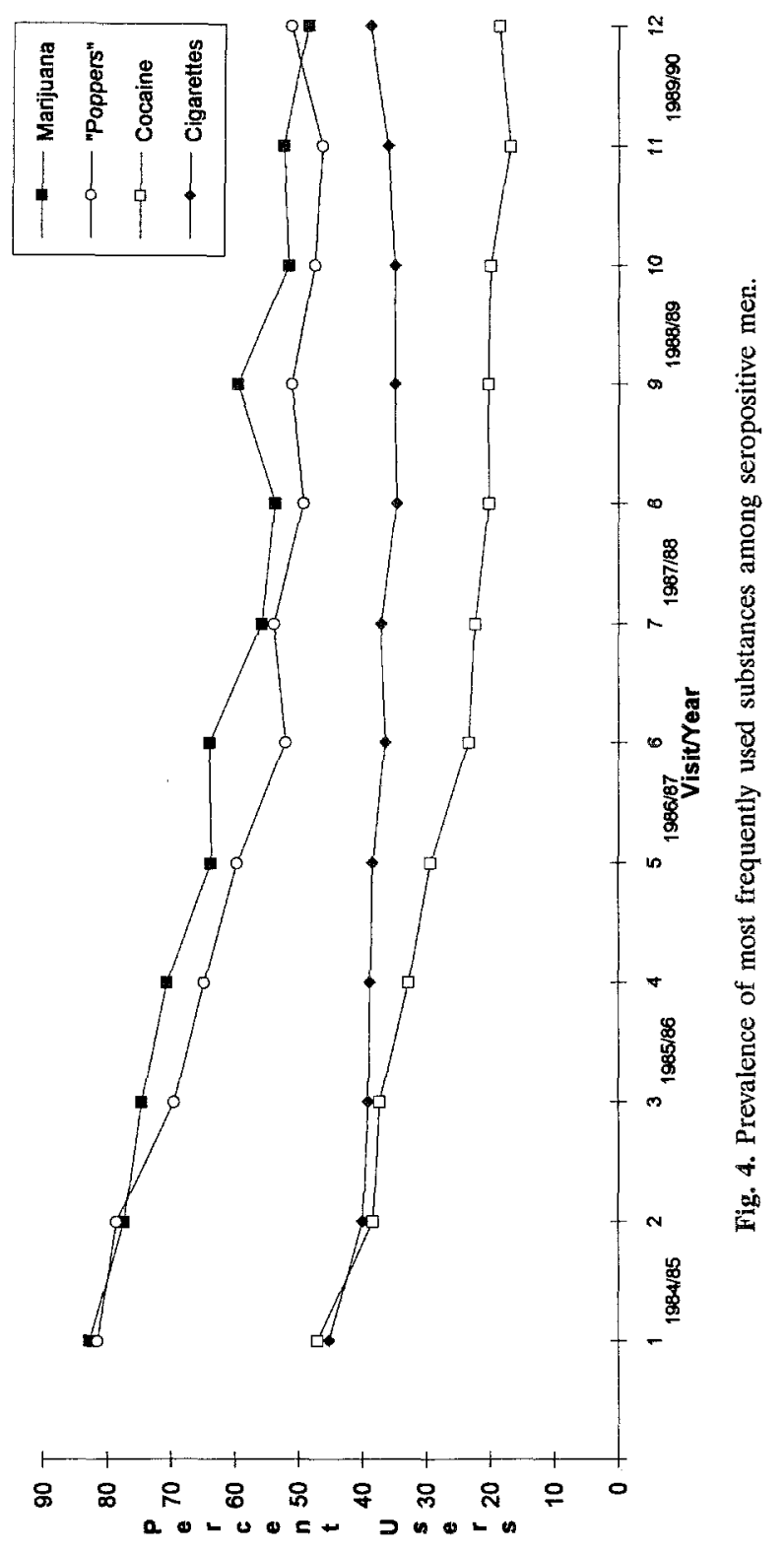




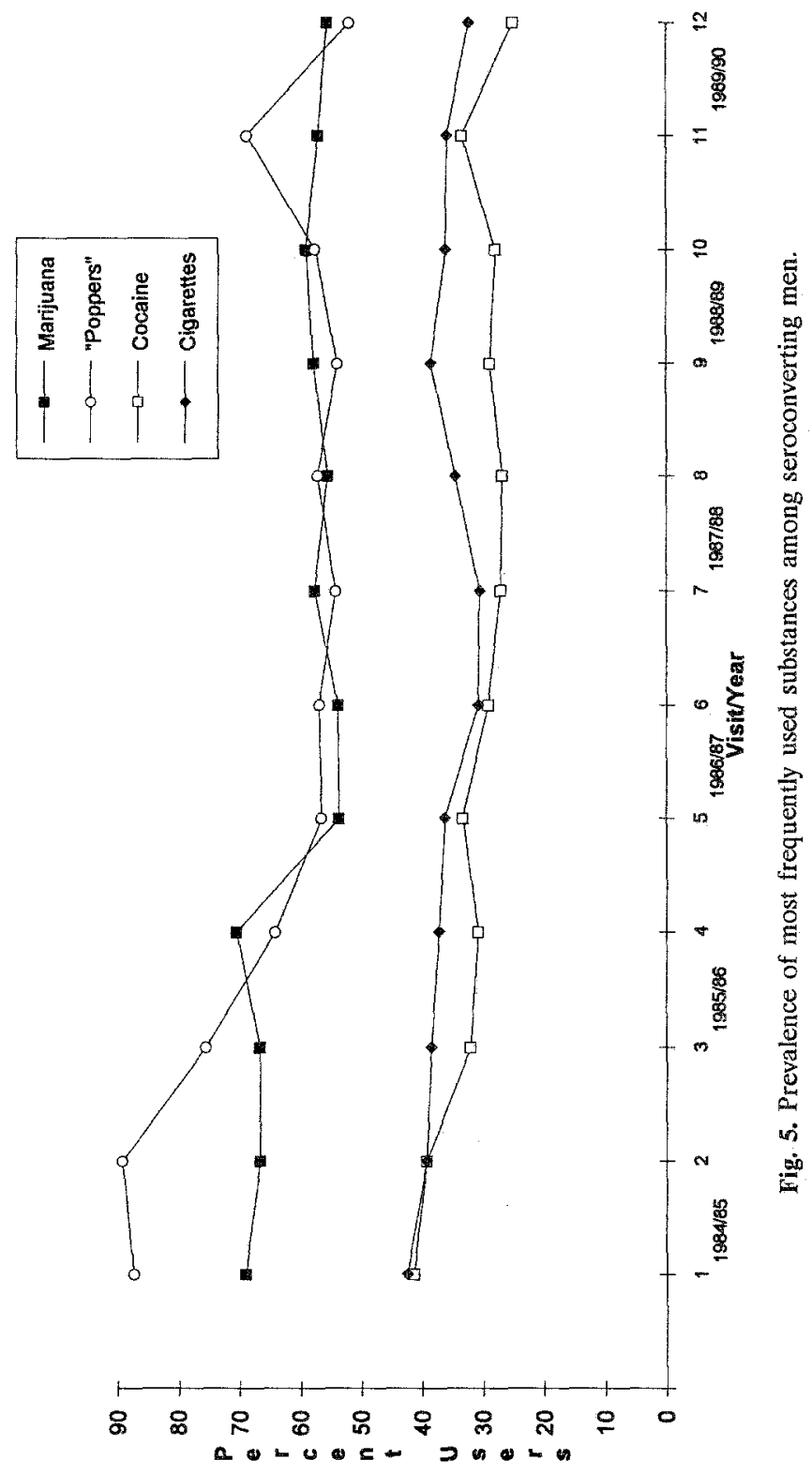


increasing risk subgroup $(n=37$ or $21 \%)$ with the stable low risk $(n=$ 72 or $41 \%)$ or decreasing risk $(n=44$ or $25 \%)$ subgroups revealed the following significant (by ANOVA) univariate differences: increasing risk men had significantly lower income $(p=0.005)$ and educational $(p=0.013)$ levels than stable low risk men (Fig. 6); increasing risk men had significantly higher levels of depressive symptomatology on the BSI than either stable low $(p=0.02)$ or decreasing $(p=0.024)$ risk men and greater levels of CES-D symptomatology than stable low risk $(p=0.008)$ men (Fig. 7); increasing risk men reported greater use of denial-fatalism coping strategies to deal with HIV-related problems than either stable low $(p=0.011)$ or decreasing risk $(p=0.019)$ men (Fig. 8); and increasing risk men reported lower levels of perceived available social support and validation and higher levels of social conflict with an isolation from their social networks than either the stable low ( $p=0.008$ to 0.03 ) or the decreasing risk ( $p=0.018$ to 0.035 ) subgroups (Fig. 9). When the same analysis was performed for the sample of nondisclosed V3-6 panel men, no significant (by ANOVA) univariate differences in psychosocial predictors were observed contrasting the various behavior change subgroups (data not shown).

These results suggest that psychosocial differences might exist between the disclosed men in the increasing risk subgroup and men in the stable low and decreasing risk subgroups. Furthermore, the fact that the observed differences were present only in the disclosed men suggested a possible "disclosure effect." When all of the increasing risk men were contrasted with all other men in the V3-6 panel using a disclosure-weighting factor, three disclosure-associated predictors-increased BSI depression, greater use of denial-fatalism coping, and lower reported collective gay orientation coping-were statistically associated (Wald's test $>1.96$ for $\alpha=5 \%$ ) with being in the increasing risk group. When the same set of analyses were performed using the psychosocial predictor without the disclosure-weighting factor, these statistical differences were no longer present. Testing these three disclosure-associated psychosocial predictors of increasing risk in multivariate logistic models, only denial-fatalism coping was significant in all models. No significant effect was observed for HIV-1 serostatus when it was included in any of the multivariate behavior change models.

\section{DISCUSSION AND CONCLUSIONS}

Taken together, the set of studies from the Chicago MACS/CCS cohort investigations reported here indicate that during a period of overall reduction in sexual risk and recreational drug use activities, there was actually a diversity of behavioral responses to HIV-1 antibody test result dis- 

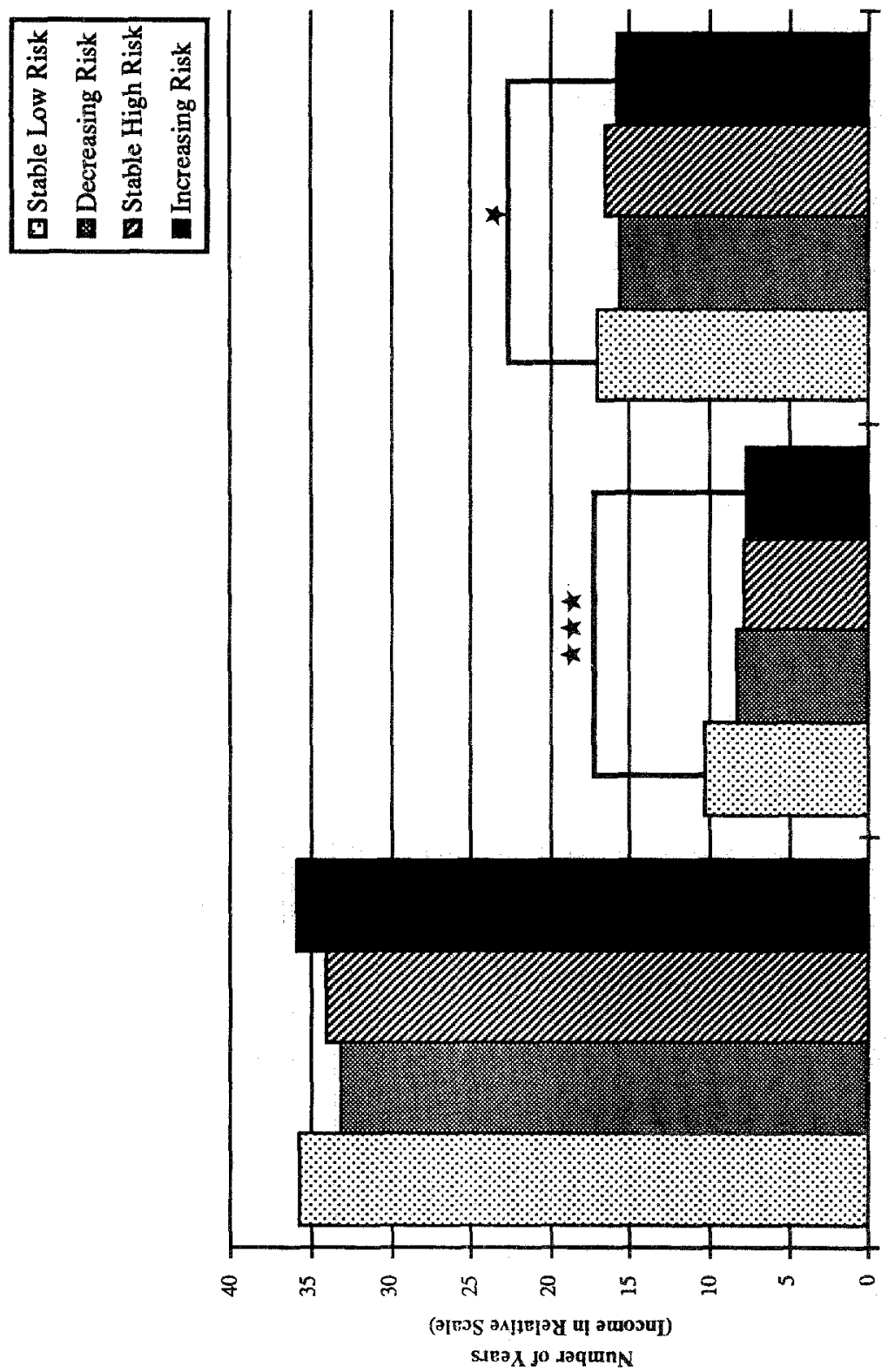

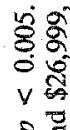

․를

몽

可

고

$\checkmark 3$

08

110

*

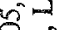

.

$\checkmark \mathrm{g}$

$0 \Omega$

11

* 氖

它

造

过

它

$=\frac{3}{0}$

$\infty$

需

品

용

उᄋ

范

药

양 प $\frac{\infty}{\circ}$ \% 곤 8

25

छี

동.

용

$5 \equiv$

$\triangle$ \&

$6=$

0. 33 

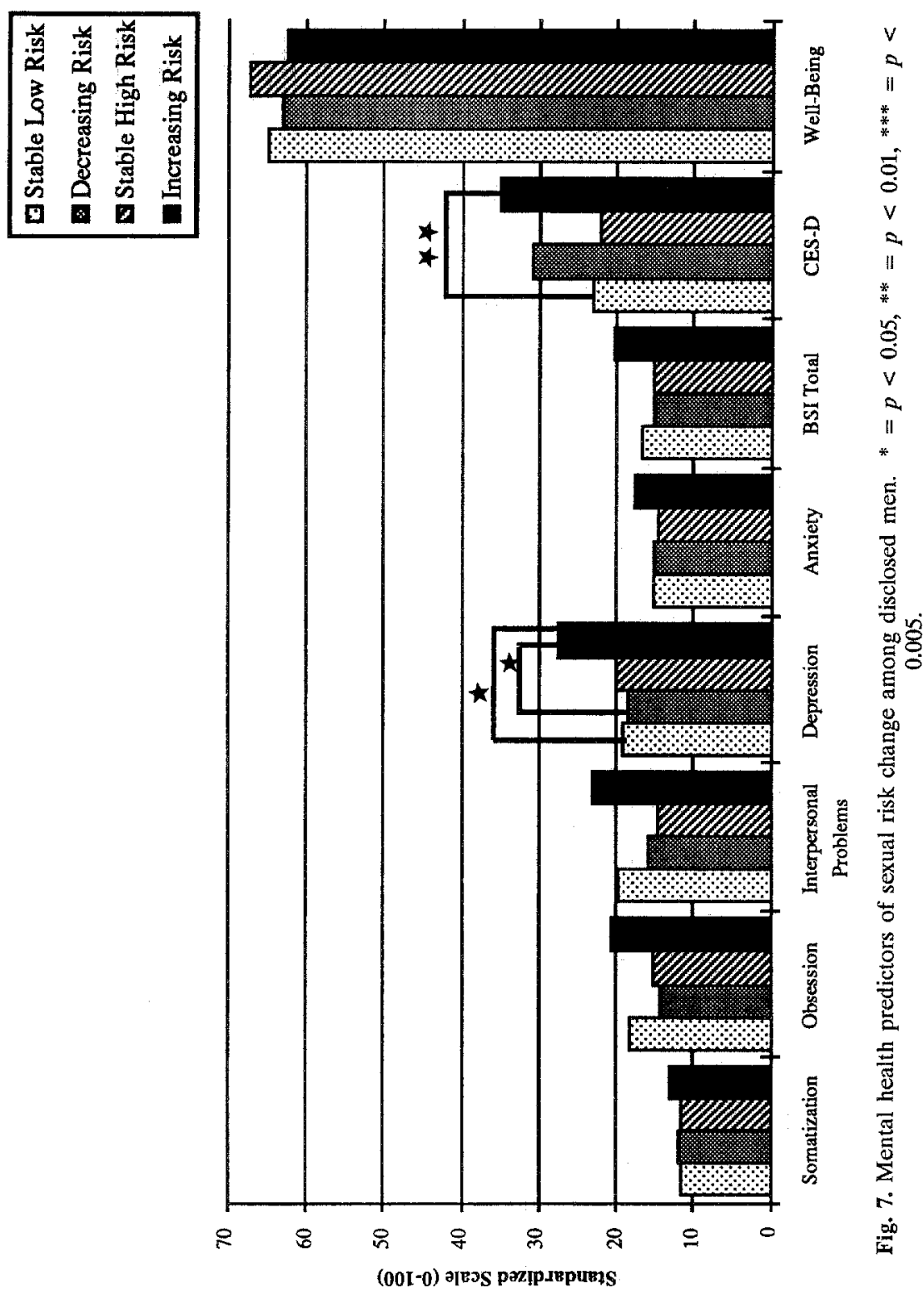


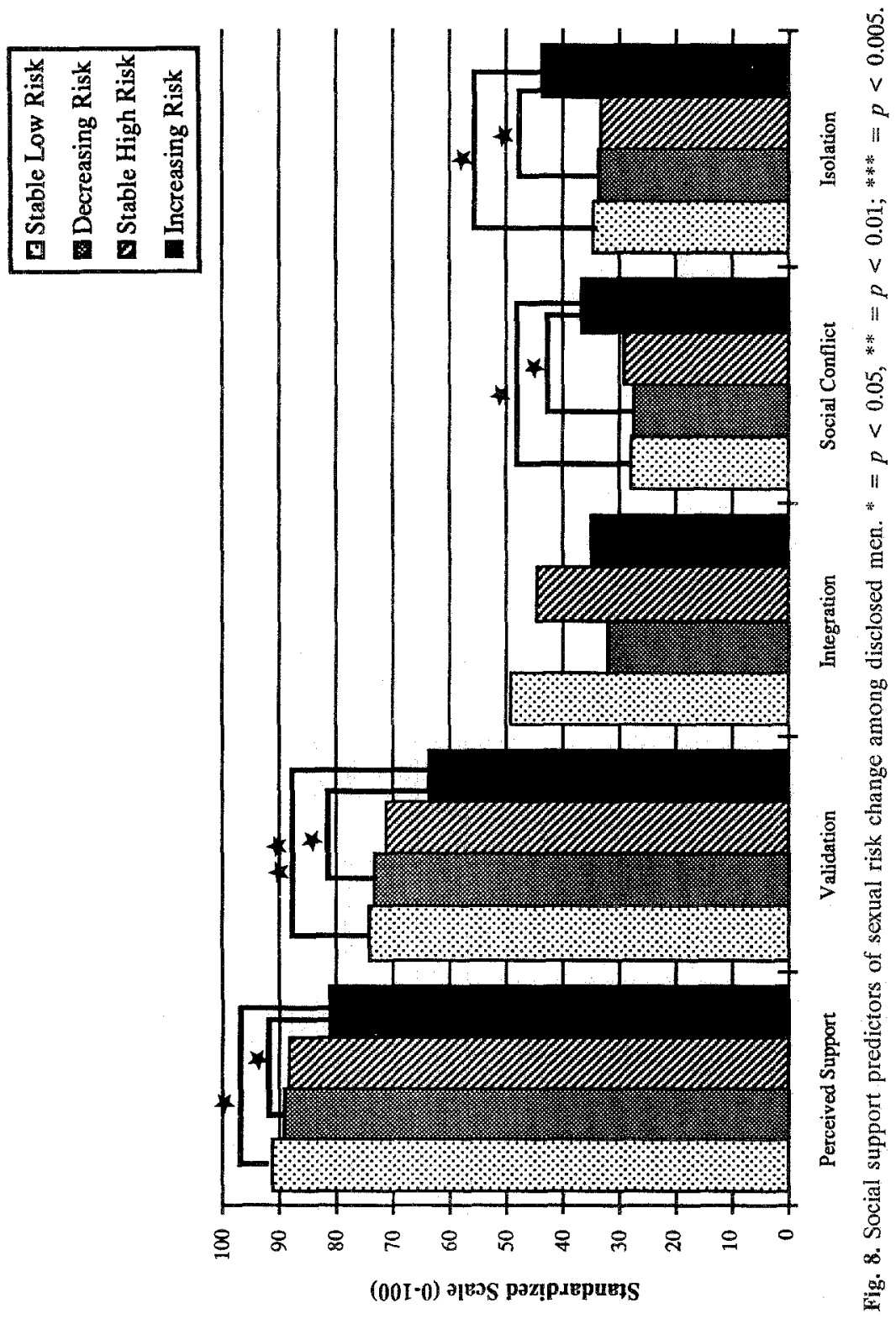




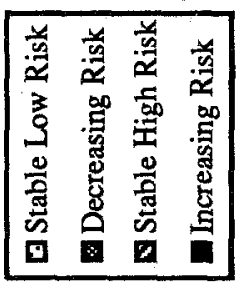

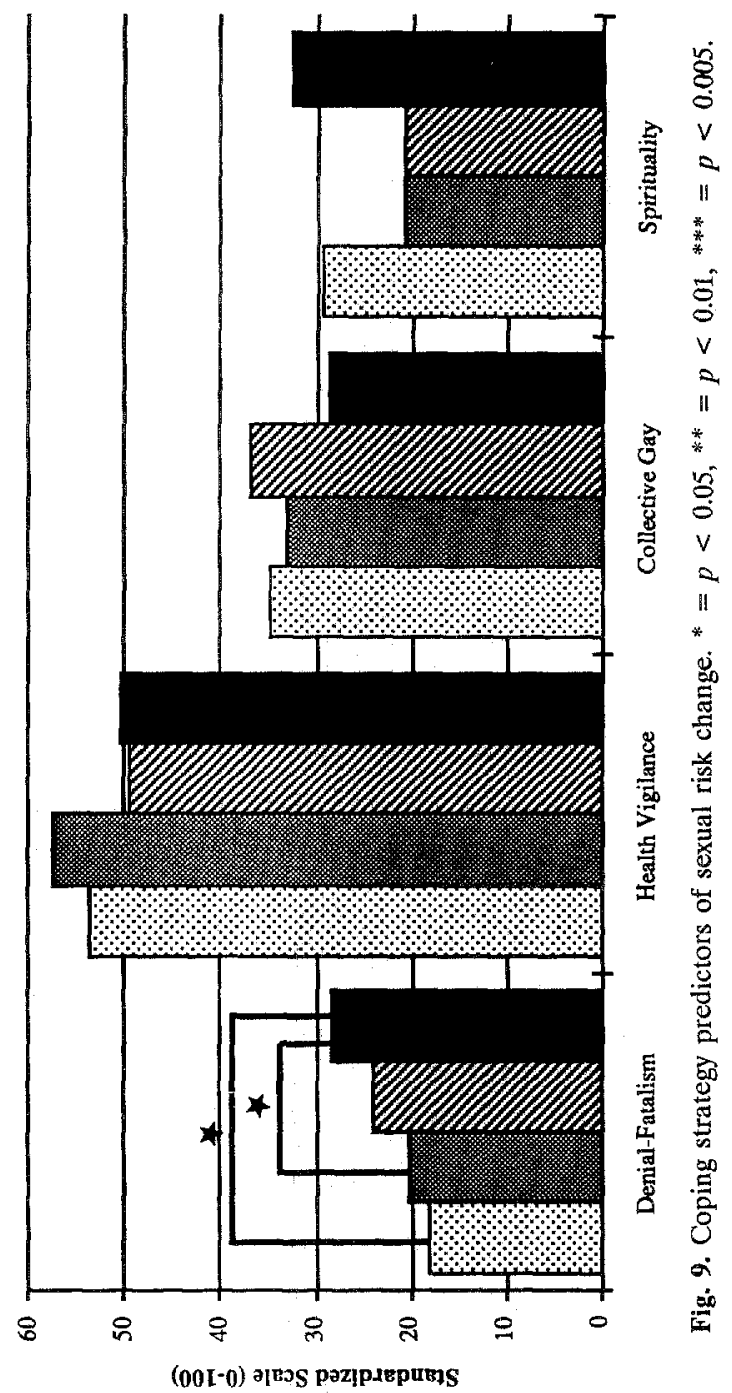


closure and counseling. In addition, the subgroup of men who reported increased risk-taking in anal receptive sexual practices subsequent to learning their HIV antibody status differed from the men reporting either stable low or decreasing patterns of RAS behavior on a number of predisclosure measures of mental health (depression), coping (denial-fatalism) and satisfaction with their social support network. In addition to considering the HIV prevention implications of these results, several important methodological issues and limitations to the generalizability of these findings warrant discussion.

First, the special nature of the Chicago MACS/CCS cohort precludes generalization of these findings to all gay or bisexual men. In particular, this cohort lacks adequate numbers of minority men to examine racial or ethnic differences in sexual or drug use behavioral change or response to HIV-1 counseling and testing. In addition, the voluntary test disclosure situation which exists for members of this cohort is not generally available to persons considering HIV-1 antibody testing. However, the relatively homogeneous nature of the Chicago MACS/CCS cohort-in terms of race, age range, sexual orientation, income, and education-can be considered an advantage for examining the effects of specific events or stressful circumstances in an otherwise shared set of social circumstances. All of the men in the cohort were sufficiently aware of the threat of AIDS to volunteer for participation in a highly demanding and intrusive set of studies in 1984, a time when the number of AIDS cases in Chicago was under 200 and large segments of the homosexually active male population did not view themselves as being at risk (Joseph et al., 1984). Thereafter, they have returned for all or most of their semiannual MACS/CCS assessment visits and have received information about AIDS, the HIV-1 antibody test, and recommendations for safer sex through their study participation. Thus, to the degree possible in any volunteer study of adult sexual behavior, this cohort can be considered to share a common level of concern about AIDS at the onset of the study and a common experience of the subsequent epidemic circumstances.

Historically, at the time of the HIV-1 serostatus disclosure study (mid1985 to mid-1987), HIV-1 antibody testing was considered experimental and many gay community opinion leaders were actively dissuading men from being tested. Fear of an adverse psychological reaction to a positive test result, perceived discrimination against seropositive persons, and lack of available treatments for asymptomatic HIV-infected persons were the most frequently cited reasons why only one third of the Chicago MACS/CCS cohort requested their antibody test results during the first 2 years that they were available (Soucey et al., 1987). Although fear of distress and discrimination are still concerns, the availability of early interventions for both the medical and psychosocial consequences of HIV infection have significantly 
shifted the risk:benefit considerations of HIV-1 counseling and testing for all at risk persons.

Keeping these methodological and historical considerations in mind, the results presented here identify a subgroup of gay/bisexual men who, at the time they sought HIV-1 antibody test results, reported high levels of denial-fatalism coping, elevated depression symptoms, and inadequate social support and who subsequently lapsed in terms of unprotected RAS in contrast to the majority of men who either adopted safer sexual behaviors or remained at low risk. The combination of inadequate social support with denial/fatalistic coping behaviors and depressed affect would be expected to identify individuals at risk of major depression or suicide. In fact, $40 \%$ of the disclosed men in the increasing sexual risk subgroup indicated suicidal ideation at the predisclosure CCS evaluation; this was twice the percentage of reported suicidal ideation reported by any of the other subgroups of disclosed or nondisclosed men. In these men, the behavioral response to learning their HIV serostatus of increased RAS risk behavior may itself be considered a form of avoidant coping or suicidal behavior.

An additional interpretation of these findings is that the increasing risk subgroup of disclosed men were less able to access and utilize the psychosocial and medical services necessary for a beneficial behavioral response to HIV counseling and testing (Coates et al., 1988; Jacobsen et al., 1990; Ostrow, 1990; Higgins et al., 1991). That these men were also less educated or affluent than the rest of the cohort supports the suggestion that they might have had limited access to biopsychosocial services at the time of learning their HIV-1 serostatus.

These findings could be used to develop mechanisms to screen gay or bisexual men seeking HIV-antibody counseling and testing to identify men at increased risk of an adverse behavioral outcome. It would be feasible to incorporate a brief interview into pretest counseling which assessed depression symptomatology, perceive social integration and adequacy of social supports, anticipated behavioral responses to a negative or positive test result, and coping strategies utilized for HIV-related stressors. Men reporting elevated depressive symptomatology, inadequate social integration or support, predominantly avoidant or denial coping styles, or anticipating an adverse behavioral reaction to the testing process could be targeted for more intensive pretest counseling and comprehensive posttest psychosocial care. Such screening and targeted intervention strategies could potentially identify those persons at risk of suicidal and/or unsafe sexual behavior responses to HIV-1 testing and greatly increase the cost-effectiveness of relatively expensive biopsychosocial interventions. In addition, selective targeting of intensive posttest counseling could improve the utilization of increasingly scarce men- 
tal health care resources while more effectively achieving the original intended HIV prevention goals of HIV-antibody testing.

\section{REFERENCES}

Adib, S. M., Joseph, J. G., Ostrow, D. G., and James, S. A. (1991). Predictors of relapse in sexual practices. AIDS Educ. Prevent. 3: 293-304.

Adib, S. M., Ostrow, D. G., and Joseph, J. G. (1992). Longitudinal patterns of sexual behavior change and relapse in the Chicago MACS/CCS cohort. VIII International Conference on AIDS, Amsterdam, The Netherlands 19-24 July, Poster Abstract PoB3806, p. B225.

Beltran, E. D., Ostrow, D. G., and Joseph, J. (1993). Predictors of sexual behavior change among men requesting their HIV-1 antibody status: The Chicago MACS/CCS cohort of homosexual/bisexual men, 1985-1986. AIDS Educ. Prevent. 5: 185-195.

Cox, D. R. (1972). Regression models and life tables. J. Roy. Statist. Soc. Ser. B 34: 187-220.

Clark, W. B., and Midanik, L. (1982). Alcohol use and alcohol problems among U.S. adults: Results of the 1979 national survey. In Alcohol and Health: Alcohol Consumption and Related Problems (Monogr. No. 1), National Institute of Alcohol Abuse and Alcoholism, United States Public Health Service, Washington, DC.

Coates, T. J., Stall, R. D., Kegeles, S. M., Lo, B., Morin, S. F., and McKusick, L. (1988). AIDS antibody testing: Will it stop the AIDS epidemic? Will it help people infected with HIV? Am. Psychol. 43: 859-864.

Derogatis, L. R., Lipman, R. S., Richels, K., Uhlenhuth E. H., and Covi L. (1974). The Hopkins Symptom Checklist (HSCL): A self-report symptom inventory. Behav. Sci. 19: 1-15.

Detels, R., English, P., Visscher, B. R. Jacobson, L., Kingsley, L. A., Chmiel, J. S., Dudley, J. P., Eldred, L. J., and Ginzburg, H. M. (1989). Seroconversion, sexual activity, and condom use among 2915 HIV seronegative men followed for up to 2 years. J. Acquired Immune Deficiency Syndrome 2: 77-83.

Emmons, C., Joseph, J. G., Wortman, C. B., Montgomery, S. B., and Ostrow, D. G. (1986). Psychosocial predictors of reported behavior change in homosexual men at risk of AIDS. Health Educ. Quart. 13: 331-345.

Harrell, F. E. (1986). The logistic procedure. In SUGI Supplemental Library User's Guide, SAS Institute, Cary, NC.

Higgins, D. L., Galavotti, C., O'Reilly, K. R., Schnell, D. J., Moore, M., Rugg, D. L., and Johnson, R. (1991). Evidence for the effects of HIV antibody counseling and testing on risk behaviors. J. Am. Med. Assoc. 266: 2419-2429.

Jacobsen, P. B., Perry, S. W., and Hirsch, D.-A. (1990). Behavioral and psychological responses to HIV antibody testing. J. Consult. Clin. Psychol. 58: 31-37.

Johnson, R. W., Ostrow, D. G., and Joseph, J. G. (1990). Educational strategies for prevention of sexual transmission of HIV. In Ostrow, D. G. (ed.), Behavioral Aspects of AIDS, Plenum Press, New York, pp. 43-70.

Joseph, J. G., Adib, S. M., Koopman, J. S., and Ostrow, D. G. (1990). Behavioral change in longitudinal studies: Adoption of condom use by homosexual/bisexual men. Am. J. Public Health 80: 1513-1514.

Joseph, J. G., Emmons, C. A., Kessler, R. C., Wortman, C. B., O'Brien, K., Hocker, W. T., and Shaefer, C. (1984). Coping with the threat of AIDS. Am. Psychol. 39: 1297-1302.

Joseph, J. G., Kessler, R. C., Wortman, C. B., Kirscht, J. P., Tal, M., Caumartin, S., Eshleman, S., and Eller, M. (1989). Are there psychological costs associated with changes in behavior to reduce AIDS risk? In Mays, V. M., Albee, G. W., and Schneider, S. F. (eds.), Primary Prevention of Aids, Sage, Newbury Park, CA, pp. 209-224.

Joseph, J. G. Montgomery, S. B., Emmons, C. A., Kessler, R. C., Ostrow, D. G., Wortman, C. B., O'Brien, K., Eller, M., and Eshleman, S. (1987). Magnitude and determinants of behavioral risk reduction: Longitudinal analysis of a cohort at risk for AIDS. Psychol. Health 1: 73-96. 
Kaslow, R., Ostrow, D. G., Detels, R., Phair, J. P., Polk, B. F., and Rinaldo, C. R. (1987). The Multicenter AIDS Cohort Study (MACS): Rationale, organization and selected characteristics of the participants. Am. J. Epidemiol. 126: 310-316.

Kingsley, L. A., Detels, R., Kaslow, R., Polk, B. F., Rinaldo, C. R., Jr., Chmiel, J., Detre, K., Kelsey, S. F., Odaka, N., and Ostrow, D. (1987). Risk factors for seroconversion to Human Immunodeficiency Virus among male homosexuals. Results from the Multicenter AIDS Cohort Study. Lancet 1: 345-348.

Kingsley, L. A., Zhou, S. J., Bacellar, H., Rinaldo, C. R., Jr., Chmiel, J., Detels, R., Saah, A., VanRaden, M., Ho, M., and Munoz, A. (1991). Temporal trends in Human Immunodeficiency Virus Type 1 seroconversion 1984-1989: A report from the Multicenter AIDS Cohort Study (MACS). Am. J. Epidemiol. 134: 331-339.

Kleinbaum, D. G., Jupper, L. L., and Morgenstern, H. (1982). Epidemiologic Research. Principles and Quantitative Methods, Von Nostrand Reinhold, New York.

Lewis-Beck, M. S. (1980). Applied Regression in the Quantitative Applications in the Social Sciences Series, Sage, Beverly Hills, pp. 54-56.

Mays, V. M., Albee, G. W., and Schneider, S. F. (1989). Primary Prevention of AIDS. Psychological Approaches, Sage, London.

McCarthy, C. R. (1988). Policy on Informing Those Tested for HIV Serostatus. NIH Office of Protection of Research Risk Reports, Washington, DC., June 10.

Montgomery, S. B., Joseph, J. G., Becker, M. H., Ostrow, D. G., Kessler, R. C., and Kirscht, J. P. (1989). The Health Belief Model in understanding compliance with preventive recommendations for AIDS: How useful? AIDS Educ. Prevent. 1: 303-323.

O'Brien, K., Wortman, C. B., Kessler, R. C., and Joseph, J. G. (1993). Social relationships of men at risk for AIDS. Soc. Sci. Med. 36: 1161-1167.

Ostrow, D. G. (1989a). AIDS prevention through effective education. Daedalus 118: 229-254.

Ostrow, D. G. (1989b). Risk reduction for transmission of Acquired Immunodeficiency Syndrome Virus in high risk communities. Psychiatr. Med. 7(2): 79-96.

Ostrow, D. G. (1990). Psychiatric aspects of HIV infection. In Current Concepts Monographs, Upjohn Publications, Kalamazoo, MI.

Ostrow, D. G., Eller, M., and Joseph, J. G. (1988). Epidemic control measures for AIDS: A psychosocial and historical discussion of policy alternatives. In Corless, I. B., and Pittmann-Lindeman, M. (eds.), AIDS: Principles, Practices, and Policies, Hemisphere, Washington, DC, pp. 19-31.

Ostrow, D. G., Joseph, J. G., Beltran, E., Chmiel, J., and Phair, J. (1989a). Characteristics and responses of men seeking HIV antibody test results. Proc. V Int. Conf. AIDS, p. 877.

Ostrow, D. G., Joseph, J. G., Kessler, R., Soucy, J., Tal, M., Eller, M., Chmiel, J., and Phair, J. P. (1989b). Disclosure of HIV antibody status: behavioral and mental health correlates. AIDS Educ. Prevent. 1: 1-11.

Ostrow, D. G., VanRaden, M. J., Fox, R., Kingsley, L. A., Dudley, J., Kaslow, R. A., and the Multicenter AIDS Cohort Study (MACS). (1990). Recreational drug use and sexual behavior change in a cohort of homosexual men. AIDS 4: 759-765.

Soucey, J., Ostrow, D. G., and Joseph, J. G. (1987). HIV antibody disclosure and behavior change. Proc. Am. Psychiat. Assoc. 140th Ann. Meeting, Chicago, IL, p. 101.

Weiss, S. H., Goedert, J. J., Sarngadharan, M. G., Bodner, A. J., Gallo, R. C., and Blattner, W. A. (1985). Screening test for HTLV-III (AIDS agent) antibodies: Specificity, sensitivity and applications. J. Am. Med. Assoc. 253: 221-225. 